\title{
Robust Variance-Constrained Filtering for A Class of Nonlinear Stochastic Systems with Missing Measurements
}

\author{
Lifeng Ma, Zidong Wang*, Jun Hu, Yuming Bo and Zhi Guo
}

\begin{abstract}
This paper is concerned with the robust filtering problem for a class of nonlinear stochastic systems with missing measurements and parameter uncertainties. The missing measurements are described by a binary switching sequence satisfying a conditional probability distribution, and the nonlinearities are expressed by the statistical means. The purpose of the filtering problem is to design a filter such that, for all admissible uncertainties and possible measurements missing, the dynamics of the filtering error is exponentially mean-square stable, and the individual steady-state error variance is not more than prescribed upper bound. A sufficient condition for the exponential mean-square stability of the filtering error system is first derived and an upper bound of the state estimation error variance is then obtained. In terms of certain linear matrix inequalities (LMIs), the solvability of the addressed problem is discussed and the explicit expression of the desired filters is also parameterized. Finally, a simulation example is provided to demonstrate the effectiveness and applicability of the proposed design approach.
\end{abstract}

\section{Keywords}

Nonlinear systems; Stochastic systems; Robust filtering; Variance constraints; Missing measurements.

\section{INTRODUCTION}

For several decades, filtering techniques have been playing an important role in many branches of signal processing such as target tracking [2]. A number of filtering approaches, including Kalman filtering, $H_{\infty}$ filtering and robust filtering, have been proposed in the literature, most of which are under the assumption that the measurements always contain true signals corrupted by the noises, see e.g. $[4,6,7,10,11,16,17,19$, 20,26,31,34]. However, in real-world applications, the measurements may contain missing measurements (or incomplete observations) due to various reasons such as high maneuverability of the tracked targets, sensor temporal failures or network congestion [21].

Because of its clear engineering insights, in the past few years, the filtering problem with missing measurements has received much attention. For linear stochastic systems, the related work was started in [15, 22] where the missing data was modeled as a binary switching sequence specified by a conditional probability distribution. Based on this model for observations missing, some results have recently been reported on the filtering problems for linear stochastic systems, see [12,13,28-30] for some examples. Specifically, in [28], a filter for stochastic uncertain systems has been designed with prescribed error variance constraints. The finite-horizon robust filtering problem has been considered in [29] for discrete-time stochastic systems with

This work was supported in part by the Engineering and Physical Sciences Research Council (EPSRC) of the U.K. under Grant GR/S27658/01, the Royal Society of the U.K. and the Alexander von Humboldt Foundation of Germany.

L. Ma, Y. Bo and Z. Guo are with the School of Automation, Nanjing University of Science and Technology, Nanjing 210094, China.

Z. Wang are with the Department of Information Systems and Computing, Brunel University, Uxbridge, Middlesex, UB8 3PH, United Kingdom. (Email: Zidong.Wang@brunel.ac.uk)

$\mathrm{J}$. $\mathrm{Hu}$ is with the Space Control and Inertial Technology Research Center, Harbin Institute of Technology, Harbin 150001, China.

* Corresponding author. 
probabilistic missing measurements subject to norm-bounded parameter uncertainties. Very recently, a robust $H_{\infty}$ filtering problem has been coped with in [30] for uncertain time-delay systems with probabilistic observation missing.

On the other hand, it is quite common in practical engineering that, for a class of filtering problems such as the tracking of a maneuvering target, the performance objectives are naturally described as the upper bounds on the error variances of estimation $[19,26,28,34]$. This gives rise to the so-called variance-constrained filtering problem, which has been motivated from the well-known covariance control theory $[5,18]$. Note that the variance-constrained filtering or control theory has been extensively investigated in a variety of practical situation $[3,35]$. As mentioned in [28], the specified variance constraints may not be minimal, but should meet given engineering requirements. Therefore, after assigning to the filtering error dynamics a specified variance upper bound, there remains much freedom which can be used to attempt to directly achieve other desired performance requirements, but the traditional optimal (robust) Kalman filtering methods may not have such an advantage.

It should be pointed out that, almost all the aforementioned results concerning variance-constrained filtering have been concerned with linear systems only, and the corresponding literature for nonlinear systems has been very few, due primarily to the difficulty in analyzing the steady-state estimation error covariance for nonlinear systems. In [34], an LMI approach has been proposed to deal with robust $H_{2}$ filtering problems for a class of stochastic nonlinear systems, but the variance constraints have not been explicitly taken into account. Up to now, to the best of authors' knowledge, in the presence of probabilistic measurements missing, the filtering problem for nonlinear stochastic systems with error variance constraints has not been investigated yet, and therefore remains open and challenging. It is, therefore, the purpose of this paper to shorten such a gap by investigating the robust filtering problem for a class of nonlinear stochastic systems with missing measurements and variance constraints.

In this paper, we model the missing measurements by a Bernoulli distributed white sequence with a known conditional probability distribution. Based on this model, the robust variance-constrained filtering problem is addressed for a class of nonlinear stochastic systems with missing measurements. We aim at designing a filter such that, for all parameter uncertainties and possible measurements missing, 1) the filtering error system is exponentially mean-square stable and 2) the variance of the estimation error for individual state is not more than prescribed upper bound. It is shown that the solvability of the addressed filtering problem can be expressed as the feasibility of a certain set of LMIs, and the explicit expression of the desired robust filters is also derived. A simulation numerical example is provided to illustrate the usefulness of the proposed design approach. The main contributions of this paper are summarized as follows: 1) a new filtering problem is studied for the stochastic systems with both stochastic nonlinearities and measurements missing phenomenon; and 2) a new error variance performance is taken into consideration for the addressed stochastic nonlinear systems with missing measurements.

The rest of this paper is arranged as follows. Section II formulates the robust variance-constrained filter design problem for uncertain nonlinear stochastic discrete-time systems. In Section III, the exponential meansquare stability of the filtering error system and the individual variance constraints of the estimation error are analyzed separately. The solution of the robust filter design problem is given in terms of a certain set of LMIs in Section IV. In Section V, an illustrative numerical example is provided to show the effectiveness and usefulness of the proposed approach. Section 6 gives our conclusions.

Notation The following notation will be used in this paper. $\mathbb{R}^{n}$ and $\mathbb{R}^{n \times m}$ denote, respectively, the $n$ dimensional Euclidean space and the set of all $n \times m$ matrices, and $\mathbb{I}^{+}$denotes the set of nonnegative integers. The notation $X \geq Y$ (respectively $X>Y$ ), where $X$ and $Y$ are symmetric matrices, means that $X-Y$ is positive semi-definite (respectively positive definite). $\operatorname{Var}\left\{x_{i}\right\}$ means the variance of $x_{i} . \mathbb{E}\{x\}$ stands for the expectation of stochastic variable $x$ and $\mathbb{E}\{x \mid y\}$ for the expectation of $x$ conditional on $y$. The superscript 
" $T$ " denotes the transpose. $\rho(A)$ means the spectral radius of matrix $A$, while $\operatorname{tr}(A)$ is the trace of matrix $A$. $\otimes$ stands for the Kronecker product of matrices. $\operatorname{diag}\left\{F_{1}, F_{2}, \ldots\right\}$ denotes a block diagonal matrix whose diagonal blocks are given by $F_{1}, F_{2} \ldots$ The symbol "*" in a matrix means that the corresponding term of the matrix can be obtained by symmetric property.

\section{Problem Formulation}

Consider the following uncertain discrete-time nonlinear stochastic system:

$$
x_{k+1}=(A+\Delta A) x_{k}+f\left(x_{k}\right)+B \omega_{k},
$$

with the measurement equation

$$
y_{k}=\gamma_{k}\left(C x_{k}+g\left(x_{k}\right)\right)+D \omega_{k}
$$

where $x_{k} \in \mathbb{R}^{n}$ is the state, $y_{k} \in \mathbb{R}^{m}$ is the measured output, and $A, B, C, D$ are known constant matrices with appropriate dimensions. $\omega_{k} \in \mathbb{R}^{n}$ is a zero mean Gaussian white noise sequence with covariance $W>0$. $\Delta A$ is a real-valued perturbation matrix that represents parametric uncertainty being of the following form:

$$
\Delta A=H F E, \quad F F^{\mathrm{T}} \leqslant I,
$$

where $H$ and $E$ are known constant matrices with appropriate dimensions. The uncertainties in $\Delta A$ are said to be admissible if (3) holds. The stochastic variable $\gamma_{k} \in \mathbb{R}$ is a Bernoulli distributed white sequence taking values on 0 and 1 with

$$
\operatorname{Prob}\left\{\gamma_{k}=1\right\}=\mathbb{E}\left\{\gamma_{k}\right\}:=\bar{\gamma},
$$

where $\bar{\gamma}$ is a known positive constant, and $\gamma_{k} \in \mathbb{R}$ is assumed to be independent of both $w_{k}$ and the system initial state $x_{0}$. Therefore, we have

$$
\begin{aligned}
& \text { Prob }\left\{\gamma_{k}=0\right\}=1-\bar{\gamma}, \\
& \sigma_{\gamma}^{2}:=\mathbb{E}\left\{\left(\gamma_{k}-\bar{\gamma}\right)^{2}\right\}=(1-\bar{\gamma}) \bar{\gamma} .
\end{aligned}
$$

Remark 1: Notice that the parameter uncertainty only enters into the system matrix $A$. However, it is worth pointing out that, within the same framework to be developed, we can also consider the case when the uncertainties exist in the output equation. The reason why we discuss the system (1)-(2) is to make our theory more understandable and to avoid unnecessarily complicated notations.

The nonlinear stochastic functions $f\left(x_{k}\right)$ and $g\left(x_{k}\right)$ are assumed to have the following first moments for all $x_{k}$ :

$$
\mathbb{E}\left\{\left[\begin{array}{l}
f\left(x_{k}\right) \\
g\left(x_{k}\right)
\end{array}\right] \mid x_{k}\right\}=0
$$

with the covariance given by

$$
\mathbb{E}\left\{\left[\begin{array}{l}
f\left(x_{k}\right) \\
g\left(x_{k}\right)
\end{array}\right]\left[\begin{array}{ll}
f^{\mathrm{T}}\left(x_{j}\right) & g^{\mathrm{T}}\left(x_{j}\right)
\end{array}\right] \mid x_{k}\right\}=0, \quad k \neq j
$$

and

$$
\mathbb{E}\left\{\left[\begin{array}{l}
f\left(x_{k}\right) \\
g\left(x_{k}\right)
\end{array}\right]\left[\begin{array}{ll}
f^{\mathrm{T}}\left(x_{k}\right) & g^{\mathrm{T}}\left(x_{k}\right)
\end{array}\right] \mid x_{k}\right\}=\sum_{i=1}^{q} \Pi_{i} x_{k}^{\mathrm{T}} \Gamma_{i} x_{k},
$$

where $\Pi_{i}$ and $\Gamma_{i}(i=1,2, \cdots, q)$ are known positive-definite matrices with following structures:

$$
\Pi_{i}=\left[\begin{array}{c}
\pi_{1 i} \\
\pi_{2 i}
\end{array}\right]\left[\begin{array}{l}
\pi_{1 i} \\
\pi_{2 i}
\end{array}\right]^{\mathrm{T}}, \quad \Gamma_{i}=\theta_{i} \theta_{i}^{\mathrm{T}},
$$


with $\pi_{1 i} \in \mathbb{R}^{n}, \pi_{2 i} \in \mathbb{R}^{m}$ and $\theta_{i} \in \mathbb{R}^{n}(i=1,2, \cdots, q)$ being known column vectors of appropriate dimensions.

Remark 2: The nonlinearity description in (6)-(8) covers several classes of well-studied nonlinear systems, for example, the system with state-dependent multiplicative noises and the system whose state's power depends on the sector-bound (or sign) of the nonlinear state function of the state, see [36].

Introduce now a new stochastic sequence

$$
\tilde{\gamma}_{k}:=\gamma_{k}-\bar{\gamma}
$$

It is easy to see that $\tilde{\gamma}_{k}$ is a scalar zero mean stochastic sequence with variance

$$
\sigma_{\tilde{\gamma}}^{2}=(1-\bar{\gamma}) \bar{\gamma}
$$

Consider the following filter for the system (1):

$$
\hat{x}_{k+1}=G \hat{x}_{k}+K\left(y_{k}-\bar{\gamma} C \hat{x}_{k}\right),
$$

where $\hat{x}_{k}$ stands for the state estimate, $G$ and $K$ are the filter parameters to be scheduled.

Define the estimation error as

$$
e_{k}=x_{k}-\hat{x}_{k},
$$

and steady-state estimation error covariance as

$$
X_{e e}:=\lim _{k \rightarrow \infty} \mathbb{E}\left\{e_{k} e_{k}^{\mathrm{T}}\right\} .
$$

Then, we obtain the following augmented system

$$
z_{k+1}=\widetilde{A} z_{k}+\widetilde{B} h\left(x_{k}\right)+\widetilde{D} \omega_{k}
$$

where

$$
\begin{aligned}
& z_{k}=\left[\begin{array}{l}
x_{k} \\
e_{k}
\end{array}\right], \quad \widetilde{A}=\left[\begin{array}{cc}
A+\Delta A & 0 \\
A+\Delta A-G-\tilde{\gamma}_{k} K C & G-\bar{\gamma} K C
\end{array}\right], \\
& \widetilde{B}=\left[\begin{array}{cc}
I & 0 \\
I & -\gamma_{k} K
\end{array}\right], \quad \widetilde{D}=\left[\begin{array}{c}
B \\
B-K D
\end{array}\right], \quad h\left(x_{k}\right)=\left[\begin{array}{l}
f\left(x_{k}\right) \\
g\left(x_{k}\right)
\end{array}\right] .
\end{aligned}
$$

Before stating our design objective, we introduce the following stability concept for the system (14).

Definition 1: [30] The system (14) is said to be exponentially mean-square stable if, with $\omega_{k}=0$, there exist constants $\zeta \geqslant 1$ and $\tau \in(0,1)$ such that

$$
\mathbb{E}\left\{\left\|z_{k}\right\|^{2}\right\} \leqslant \zeta \tau^{k} \mathbb{E}\left\{\left\|z_{0}\right\|^{2}\right\}, \quad \forall z_{0} \in \mathbb{R}^{2 n}, \quad k \in \mathbb{I}^{+} .
$$

for all admissible uncertainties and possible measurements missing.

In this paper, our objective is to design the filter (11) for the system (1) such that, for all admissible uncertainties and possible measurements missing, the following two objectives are satisfied simultaneously:

Q1) The augmented system (14) is exponentially mean-square stable;

Q2) The steady-state error variance $X_{e e}$ satisfies

$$
X_{e e}^{i} \leqslant \sigma_{i}^{2} \quad i=1,2, \cdots, n,
$$

where $X_{e e}^{i}$ stands for the steady-state variance of the $i$ th error state, and $\sigma_{i}^{2}(i=1,2, \cdots, n)$ denotes the prespecified steady-state estimation error variance constraint on the $i$ th state.

Remark 3: In engineering practice, the variance upper bounds which represent the control or estimation precision of the system state should be specified according to the actual requirements before the system 
design. For instance, in the problem of tracking maneuvering targets, the position and velocity of the target are measured in every sampling instant. However, due to the high maneuver of the tracked target, it is neither possible nor necessary to track the target in a precise way. Instead, an acceptable compromise is to keep the target within a given "window" as frequent as possible, and such a requirement can be expressed as upper bounds on the estimation error variance. In this sense, before the actual system design, we could specify the error variance upper bound according to the length and width of the required "window".

\section{STABILITY AND VARIANCE ANALYSIS}

Before giving our derivation, we first introduce some useful lemmas.

Lemma 1: [27] Let $V\left(z_{k}\right)=z_{k}^{\mathrm{T}} P z_{k}$ be a Lyapunov functional where $P>0$. If there exist real scalars $\lambda$, $\mu>0, \nu>0$ and $0<\psi<1$ such that both

$$
\mu\left\|z_{k}\right\|^{2} \leqslant V\left(z_{k}\right) \leqslant \nu\left\|z_{k}\right\|^{2}
$$

and

$$
\mathbb{E}\left\{V\left(z_{k+1}\right) \mid z_{k}\right\}-V\left(z_{k}\right) \leqslant \lambda-\psi V\left(z_{k}\right),
$$

hold, then the process $z_{k}$ satisfies

Denote

$$
\mathbb{E}\left\{\left\|z_{k}\right\|^{2}\right\} \leqslant \frac{\nu}{\mu}\left\|z_{0}\right\|^{2}(1-\psi)^{k}+\frac{\lambda}{\mu \psi}
$$

$$
\begin{aligned}
& \widehat{A}=\left[\begin{array}{cc}
A & 0 \\
A-G & G-\bar{\gamma} K C
\end{array}\right], \quad \Delta \widehat{A}=\left[\begin{array}{cc}
\Delta A & 0 \\
\Delta A & 0
\end{array}\right], \quad J=\left[\begin{array}{cc}
0 & 0 \\
\sigma_{\tilde{\gamma}} K C & 0
\end{array}\right], \\
& \widetilde{\Gamma}_{i}=\left[\begin{array}{cc}
\Gamma_{i} & 0 \\
0 & 0
\end{array}\right], \quad \widetilde{\Pi}_{i}=\left[\begin{array}{cc}
\pi_{1 i} \pi_{1 i}^{\mathrm{T}} & \pi_{1 i} \pi_{1 i}^{\mathrm{T}}-\bar{\gamma} \pi_{1 i} \pi_{2 i}^{\mathrm{T}} K^{\mathrm{T}} \\
* & \pi_{1 i} \pi_{1 i}^{\mathrm{T}}-\bar{\gamma}\left(\pi_{1 i} \pi_{2 i}^{\mathrm{T}} K^{\mathrm{T}}+K \pi_{2 i} \pi_{1 i}^{\mathrm{T}}\right)+\left(\bar{\gamma}^{2}+\sigma_{\gamma}^{2}\right) K \pi_{2 i} \pi_{2 i}^{\mathrm{T}} K^{\mathrm{T}}
\end{array}\right] .
\end{aligned}
$$

Lemma 2: Given the filter parameters $G$ and $K$. The following statements are equivalent.

1)

$$
\rho\left\{(\widehat{A}+\Delta \widehat{A})^{\mathrm{T}} \otimes(\widehat{A}+\Delta \widehat{A})^{\mathrm{T}}+J^{\mathrm{T}} \otimes J^{\mathrm{T}}+\sum_{i=1}^{q} \operatorname{st}\left(\widetilde{\Gamma}_{i}\right) \mathrm{st}^{\mathrm{T}}\left(\widetilde{\Pi}_{i}\right)\right\}<1
$$

or

$$
\rho\left\{(\widehat{A}+\Delta \widehat{A}) \otimes(\widehat{A}+\Delta \widehat{A})+J \otimes J+\sum_{i=1}^{q} \operatorname{st}\left(\widetilde{\Pi}_{i}\right) \operatorname{st}^{\mathrm{T}}\left(\widetilde{\Gamma}_{i}\right)\right\}<1 .
$$

2) There exists a positive definite matrix $P>0$ such that

$$
(\widehat{A}+\Delta \widehat{A})^{\mathrm{T}} P(\widehat{A}+\Delta \widehat{A})+J^{\mathrm{T}} P J-P+\sum_{i=1}^{q} \widetilde{\Gamma}_{i} \operatorname{tr}\left[P \widetilde{\Pi}_{i}\right]<0 .
$$

3) There exists a positive definite matrix $Q>0$ such that

$$
(\widehat{A}+\Delta \widehat{A}) Q(\widehat{A}+\Delta \widehat{A})^{\mathrm{T}}+J Q J^{\mathrm{T}}-Q+\sum_{i=1}^{q} \widetilde{\Pi}_{i} \operatorname{tr}\left[Q \widetilde{\Gamma}_{i}\right]<0 .
$$

4) The system (14) is exponentially mean-square stable.

Proof: First, it can be noticed that the main difference between this lemma and Theorem 1 of [36] is that the state matrix of the system (14) in this paper contains stochastic variables $\gamma_{k}$ and $\tilde{\gamma}_{k}$ which result from the possible measurements missing in the process of output sampling. Hence, we just need to prove the 
relationship “2) $\Rightarrow 4$ )" in order to demonstrate how we tackle the matrix involving stochastic variables in the derivation, and the rest of this lemma can be easily proved as in Theorem 1 of [36] using the techniques shown below.

2) $\Rightarrow 4$ ): Define a Lyapunov functional $V\left(z_{k}\right)=z_{k}^{\mathrm{T}} P z_{k}$ where $P>0$ is the solution to (23). Then,

$$
\begin{aligned}
& \mathbb{E}\left\{V\left(z_{k+1}\right) \mid z_{k}\right\}-V\left(z_{k}\right) \\
& =\mathbb{E}\left\{\left(\widetilde{A} z_{k}+\widetilde{B} h\left(x_{k}\right)\right)^{\mathrm{T}} P\left(\widetilde{A} z_{k}+\widetilde{B} h\left(x_{k}\right)\right) \mid z_{k}\right\}-z_{k}^{\mathrm{T}} P z_{k} \\
& =\mathbb{E}\left\{\left(z_{k}^{\mathrm{T}} \widetilde{A}^{\mathrm{T}} P \widetilde{A} z_{k}\right) \mid z_{k}\right\}+\mathbb{E}\left\{\left(h^{\mathrm{T}}\left(x_{k}\right) \widetilde{B}^{\mathrm{T}} P \widetilde{B} h\left(x_{k}\right)\right) \mid z_{k}\right\}-z_{k}^{\mathrm{T}} P z_{k} .
\end{aligned}
$$

Using the statistics of $\omega_{k}, \gamma_{k}$ and $\tilde{\gamma_{k}}$, we obtain

$$
\begin{aligned}
& \mathbb{E}\left\{\left(z_{k}^{\mathrm{T}} \widetilde{A}^{\mathrm{T}} P \widetilde{A} z_{k}\right) \mid z_{k}\right\} \\
& =\mathbb{E}\left\{z_{k}^{\mathrm{T}}\left((\widehat{A}+\Delta \widehat{A})+\tilde{\gamma}_{k}\left[\begin{array}{cc}
0 & 0 \\
-K C & 0
\end{array}\right]\right)^{\mathrm{T}} P\left((\widehat{A}+\Delta \widehat{A})+\tilde{\gamma}_{k}\left[\begin{array}{cc}
0 & 0 \\
-K C & 0
\end{array}\right]\right) z_{k}\right\} \\
& =z_{k}^{\mathrm{T}}\left((\widehat{A}+\Delta \widehat{A})^{\mathrm{T}} P(\widehat{A}+\Delta \widehat{A})+J^{\mathrm{T}} P J\right) z_{k}, \\
& \mathbb{E}\left\{\left(h^{\mathrm{T}}\left(x_{k}\right) \widetilde{B}^{\mathrm{T}} P \widetilde{B} h\left(x_{k}\right)\right) \mid z_{k}\right\} \\
& =\mathbb{E}\left\{\operatorname{tr}\left(\widetilde{B}^{\mathrm{T}} P \widetilde{B} h\left(x_{k}\right) h^{\mathrm{T}}\left(x_{k}\right)\right) \mid z_{k}\right\} \\
& =\sum_{i=1}^{q} \mathbb{E}\left\{\operatorname{tr}\left(\widetilde{B} \mathrm{~T} P \widetilde{B} \Pi_{i}\right) z_{k}^{\mathrm{T}} \widetilde{\Gamma}_{i} z_{k} \mid z_{k}\right\} \\
& =z_{k}^{\mathrm{T}} \sum_{i=1}^{q} \widetilde{\Gamma}_{i} \mathbb{E}\left\{\operatorname{tr}\left(P \widetilde{B} \Pi_{i} \widetilde{B}^{\mathrm{T}}\right)\right\} z_{k},
\end{aligned}
$$

and

$$
\widetilde{B}=\left[\begin{array}{cc}
I & 0 \\
I & -\bar{\gamma} K
\end{array}\right]+\left(\bar{\gamma}-\gamma_{k}\right)\left[\begin{array}{cc}
0 & 0 \\
0 & K
\end{array}\right]
$$

Then, we have

$$
\begin{aligned}
\widetilde{B} \Pi_{i} \widetilde{B}^{\mathrm{T}} & =\widetilde{B}\left[\begin{array}{l}
\pi_{1 i} \\
\pi_{2 i}
\end{array}\right]\left[\begin{array}{l}
\pi_{1 i} \\
\pi_{2 i}
\end{array}\right]^{\mathrm{T}} \widetilde{B}^{\mathrm{T}} \\
& =\left(\left[\begin{array}{c}
\pi_{1 i} \\
\pi_{1 i}-\bar{\gamma} K \pi_{2 i}
\end{array}\right]+\left(\bar{\gamma}-\gamma_{k}\right)\left[\begin{array}{c}
0 \\
K \pi_{2 i}
\end{array}\right]\right)\left(\left[\begin{array}{c}
\pi_{1 i} \\
\pi_{1 i}-\bar{\gamma} K \pi_{2 i}
\end{array}\right]+\left(\bar{\gamma}-\gamma_{k}\right)\left[\begin{array}{c}
0 \\
K \pi_{2 i}
\end{array}\right]\right)^{\mathrm{T}},
\end{aligned}
$$

and therefore

$$
\begin{aligned}
& \mathbb{E}\left\{\operatorname{tr}\left(P \widetilde{B} \Pi_{i} \widetilde{B}^{\mathrm{T}}\right)\right\} \\
& =\mathbb{E}\left\{\operatorname{tr}\left(P\left[\begin{array}{c}
\pi_{1 i} \\
\pi_{1 i}-\bar{\gamma} K \pi_{2 i}
\end{array}\right]\left[\begin{array}{c}
\pi_{1 i} \\
\pi_{1 i}-\bar{\gamma} K \pi_{2 i}
\end{array}\right]^{\mathrm{T}}\right)+\left(\bar{\gamma}-\gamma_{k}\right)^{2} \operatorname{tr}\left(P\left[\begin{array}{c}
0 \\
K \pi_{2 i}
\end{array}\right]\left[\begin{array}{c}
0 \\
K \pi_{2 i}
\end{array}\right]^{\mathrm{T}}\right)\right\} \\
& =\operatorname{tr}\left(P\left[\begin{array}{cc}
\pi_{1 i} \pi_{1 i}^{\mathrm{T}} & \pi_{1 i} \pi_{1 i}^{\mathrm{T}}-\bar{\gamma} \pi_{1 i} \pi_{2 i}^{\mathrm{T}} K^{\mathrm{T}} \\
* & \pi_{1 i} \pi_{1 i}^{\mathrm{T}}-\bar{\gamma}\left(\pi_{1 i} \pi_{2 i}^{\mathrm{T}} K^{\mathrm{T}}+K \pi_{2 i} \pi_{1 i}^{\mathrm{T}}\right)+\bar{\gamma}^{2} K \pi_{2 i} \pi_{2 i}^{\mathrm{T}} K^{\mathrm{T}}
\end{array}\right]\right) \\
& +\sigma_{\gamma}^{2} \operatorname{tr}\left(P\left[\begin{array}{cc}
0 & 0 \\
0 & K \pi_{2 i} \pi_{2 i}^{\mathrm{T}} K^{\mathrm{T}}
\end{array}\right]\right) \\
& =\operatorname{tr}\left(P \widetilde{\Pi}_{i}\right) .
\end{aligned}
$$


Hence,

$$
\mathbb{E}\left\{V\left(z_{k+1}\right) \mid z_{k}\right\}-V\left(z_{k}\right)=z_{k}^{\mathrm{T}}\left((\widehat{A}+\Delta \widehat{A})^{\mathrm{T}} P(\widehat{A}+\Delta \widehat{A})+J^{\mathrm{T}} P J-P+\sum_{i=1}^{q} \widetilde{\Gamma}_{i} \operatorname{tr}\left[P \widetilde{\Pi}_{i}\right]\right) z_{k}
$$

It follows from (23) that there always exists a sufficiently small scalar $\eta$ satisfying $0<\eta<\lambda_{\max }(P)$ such that

$$
(\widehat{A}+\Delta \widehat{A})^{\mathrm{T}} P(\widehat{A}+\Delta \widehat{A})+J^{\mathrm{T}} P J-P+\sum_{i=1}^{q} \widetilde{\Gamma}_{i} \operatorname{tr}\left[P \widetilde{\Pi}_{i}\right]<-\eta I,
$$

which means

$$
\mathbb{E}\left\{V\left(z_{k+1}\right) \mid z_{k}\right\}-V\left(z_{k}\right) \leqslant-\eta z_{k}^{\mathrm{T}} z_{k} \leqslant-\frac{\eta}{\lambda_{\max }(P)} V\left(z_{k}\right) .
$$

Finally, the exponential mean-square stability of (14) can be immediately obtained from Lemma 1.

Lemma 3: Given the filter parameters $G$ and $K$. If the system (14) is exponentially mean-square stable and there exists a symmetric matrix $Y$ satisfying

$$
(\widehat{A}+\Delta \widehat{A}) Y(\widehat{A}+\Delta \widehat{A})^{\mathrm{T}}+J Y J^{\mathrm{T}}-Y+\sum_{i=1}^{q} \widetilde{\Pi}_{i} \operatorname{tr}\left[Y \widetilde{\Gamma}_{i}\right]<0
$$

then $Y \geqslant 0$.

Proof: Lemma 3 can be easily proved by the Lyapunov method together with Lemma 2, hence the proof is omitted.

Now, let us proceed to deal with the error variance constraints. Defining the state covariance of system (14) by

$$
Q_{k}:=\mathbb{E}\left\{z_{k} z_{k}^{\mathrm{T}}\right\}=\mathbb{E}\left\{\left[\begin{array}{l}
x_{k} \\
e_{k}
\end{array}\right]\left[\begin{array}{l}
x_{k} \\
e_{k}
\end{array}\right]^{\mathrm{T}}\right\}:=\left[\begin{array}{cc}
X_{x x k} & X_{x e k} \\
X_{x e k}^{\mathrm{T}} & X_{e e k}
\end{array}\right],
$$

the evolution of $Q_{k}$ can be derived from the system (14) as follows:

$$
Q_{k+1}=(\widehat{A}+\Delta \widehat{A}) Q_{k}(\widehat{A}+\Delta \widehat{A})^{\mathrm{T}}+J Q_{k} J^{\mathrm{T}}+\sum_{i=1}^{q} \widetilde{\Pi}_{i} \operatorname{tr}\left[Q_{k} \widetilde{\Gamma}_{i}\right]+\widetilde{D} W \widetilde{D}^{\mathrm{T}} .
$$

Rewrite (36) in the form of stack matrices

$$
\operatorname{st}\left(Q_{k+1}\right)=\left[(\widehat{A}+\Delta \widehat{A}) \otimes(\widehat{A}+\Delta \widehat{A})+J \otimes J+\sum_{i=1}^{q} \operatorname{st}\left(\widetilde{\Pi}_{i}\right) \operatorname{st}^{\mathrm{T}}\left(\widetilde{\Gamma}_{i}\right)\right] \operatorname{st}\left(Q_{k}\right)+\operatorname{st}\left(\widetilde{D} W \widetilde{D}^{\mathrm{T}}\right) .
$$

If the system (14) is exponentially mean-square stable, it then follows from Lemma 2 that (22) holds, hence in the steady-state,

$$
\widehat{Q}:=\lim _{k \rightarrow \infty} Q_{k}=\left[\begin{array}{cc}
X_{x x} & X_{x e} \\
X_{x e}^{\mathrm{T}} & X_{e e}
\end{array}\right]
$$

exists and satisfies

$$
(\widehat{A}+\Delta \widehat{A}) \widehat{Q}(\widehat{A}+\Delta \widehat{A})^{\mathrm{T}}+J \widehat{Q} J^{\mathrm{T}}-\widehat{Q}+\sum_{i=1}^{q} \widetilde{\Pi}_{i} \operatorname{tr}\left[\widehat{Q} \widetilde{\Gamma}_{i}\right]+\widetilde{D} W \widetilde{D}^{\mathrm{T}}=0 .
$$

Based on the results we have obtained so far concerning the exponential mean-square stability as well as steady-state variance, we are now ready to cope with the addressed multiobjective filter design problem. 


\section{Robust FILTER DESigN}

In this section, an LMI method is proposed to design the robust variance-constrained filter for the uncertain nonlinear stochastic system with missing measurements. To start with, a corollary is given that combines exponential mean-square stability and the error variance upper bound constraints.

Corollary 1: Given the filter parameters $G$ and $K$. If there exits a positive definite matrix $Q>0$ such that

$$
(\widehat{A}+\Delta \widehat{A}) Q(\widehat{A}+\Delta \widehat{A})^{\mathrm{T}}+J Q J^{\mathrm{T}}-Q+\sum_{i=1}^{q} \widetilde{\Pi}_{i} \operatorname{tr}\left[Q \widetilde{\Gamma}_{i}\right]+\widetilde{D} W \widetilde{D}^{\mathrm{T}}<0
$$

holds, then the system (14) is exponentially mean-square stable and the steady-state state covariance satisfies $\widehat{Q} \leqslant Q$.

Proof: It follows from (40) that

$$
(\widehat{A}+\Delta \widehat{A}) Q(\widehat{A}+\Delta \widehat{A})^{\mathrm{T}}+J Q J^{\mathrm{T}}-Q+\sum_{i=1}^{q} \widetilde{\Pi}_{i} \operatorname{tr}\left[Q \widetilde{\Gamma}_{i}\right]<-\widetilde{D} W \widetilde{D}^{\mathrm{T}}<0
$$

which indicates from Lemma 2 that the system (14) is exponentially mean-square stable. Therefore, in the steady-state, the state covariance of (14) $\widehat{Q}$ exists and satisfies (39). Subtracting (39) from (40), we obtain

$$
(\widehat{A}+\Delta \widehat{A})(Q-\widehat{Q})(\widehat{A}+\Delta \widehat{A})^{\mathrm{T}}+J(Q-\widehat{Q}) J^{\mathrm{T}}-(Q-\widehat{Q})+\sum_{i=1}^{q} \widetilde{\Pi}_{i} \operatorname{tr}\left[(Q-\widehat{Q}) \widetilde{\Gamma}_{i}\right]<0
$$

From Lemma 3, we know that $Q-\widehat{Q} \geqslant 0$ and the proof is complete.

In the next stage, we shall present the filter designing technique for the nonlinear stochastic system in the presence of probabilistic measurements missing.

The following theorem provides an LMI approach to the addressed filter design problem for the uncertain discrete-time nonlinear stochastic system (1)-(2).

Theorem 1: Given $\sigma_{i}^{2}>0(i=1,2, \cdots, n)$. If there exist positive definite matrices $R>0, S>0$, real matrices $M, N$, a positive scalar $\varepsilon$ and positive scalars $\alpha_{i}>0(i=1,2, \cdots, q)$ such that, for all admissible parameter uncertainties and possible measurement missing, the following set of LMIs:

$$
\begin{gathered}
{\left[\begin{array}{cc}
-\alpha_{i} & \alpha_{i} \theta_{i}^{\mathrm{T}} \\
\alpha_{i} \theta_{i} & -R
\end{array}\right]<0,} \\
\Psi:=\left[\begin{array}{cc}
\Psi_{11} & \Psi_{12} \\
\Psi_{12}^{\mathrm{T}} & \Psi_{22}
\end{array}\right]<0, \\
\widehat{X}-S \leqslant 0,
\end{gathered}
$$

where

$$
\Psi_{11}=\left[\begin{array}{cccccc}
-R & 0 & R A & 0 & 0 & 0 \\
* & -S & S A-M & M-\bar{\gamma} N C & \sigma_{\tilde{\gamma}} N C & 0 \\
* & * & -R & 0 & 0 & 0 \\
* & * & * & -S & 0 & 0 \\
* & * & * & * & -R & 0 \\
* & * & * & * & * & -S
\end{array}\right]
$$




$$
\Psi_{12}=\left[\begin{array}{ccccccccc}
R \pi_{11} & \cdots & R \pi_{1 q} & 0 & \cdots & 0 & R B & R H & 0 \\
S \pi_{11}-\bar{\gamma} N \pi_{21} & \cdots & S \pi_{1 q}-\bar{\gamma} N \pi_{2 q} & \sigma_{\gamma} N \pi_{21} & \cdots & \sigma_{\gamma} N \pi_{2 q} & S B-N D & S H & 0 \\
0 & \cdots & 0 & 0 & \cdots & 0 & 0 & 0 & \varepsilon E^{\mathrm{T}} \\
0 & \cdots & 0 & 0 & \cdots & 0 & 0 & 0 & 0 \\
0 & \cdots & 0 & 0 & \cdots & 0 & 0 & 0 & 0 \\
0 & \cdots & 0 & 0 & \cdots & 0 & 0 & 0 & 0
\end{array}\right],
$$

is feasible, then there exists a filter of the form (11) such that the requirements $Q 1)$ and Q2) are simultaneously satisfied. Moreover, the desired filter can be determined by

$$
\begin{aligned}
& G=S^{-1} M, \\
& K=S^{-1} N .
\end{aligned}
$$

Proof: Assume that the matrix $Q$ has a block diagonal form as follows:

$$
Q=\left[\begin{array}{cc}
R & 0 \\
0 & S
\end{array}\right]^{-1}>0
$$

where $R>0$ and $S>0$ are both $n \times n$ real valued matrices. Now, we define new variables $\alpha_{i}>0$ $(i=1,2, \cdots, q)$ satisfying

Letting

$$
\alpha_{i}<\left(\operatorname{tr}\left[Q \widetilde{\Gamma}_{i}\right]\right)^{-1}
$$

$$
\breve{\theta}_{i}=\left[\begin{array}{c}
\theta_{i} \\
0
\end{array}\right] \in \mathbb{R}^{2 n}
$$

we have $\widetilde{\Gamma}_{i}=\breve{\theta}_{i} \breve{\theta}_{i}^{\mathrm{T}}$.

Using the property of matrix trace and Schur Complement (Lemma 3 in [28]), we have

$$
\operatorname{tr}\left[Q \widetilde{\Gamma}_{i}\right]<\alpha_{i}^{-1} \Longleftrightarrow\left[\begin{array}{cc}
-\alpha_{i} & \alpha_{i} \breve{\theta}_{i}^{\mathrm{T}} \\
\alpha_{i} \breve{\theta}_{i} & -Q^{-1}
\end{array}\right]<0
$$

Then, after transformation, (50) is equivalent to (43).

Next, we prove that (44) is equivalent to

$$
(\widehat{A}+\Delta \widehat{A}) Q(\widehat{A}+\Delta \widehat{A})^{\mathrm{T}}+J Q J^{\mathrm{T}}-Q+\sum_{i=1}^{q} \widetilde{\Pi}_{i} \alpha_{i}^{-1}+\widetilde{D} W \widetilde{D}^{\mathrm{T}}<0 .
$$

By Schur Complement, (51) is equivalent to

$$
\left[\begin{array}{cccc}
-Q+\sum_{i=1}^{q} \widetilde{\Pi}_{i} \alpha_{i}^{-1} & \widehat{A}+\Delta \widehat{A} & J & \widetilde{D} \\
\widehat{A}^{\mathrm{T}}+\Delta \widehat{A}^{\mathrm{T}} & -Q^{-1} & 0 & 0 \\
J^{T} & 0 & -Q^{-1} & 0 \\
\widetilde{D}^{\mathrm{T}} & 0 & 0 & -W^{-1}
\end{array}\right]<0 .
$$

Performing the congruence transformation by $\operatorname{diag}\left\{Q^{-1}, I, I, I\right\}$, we can see that (52) is equivalent to

$$
\left[\begin{array}{cccc}
-Q^{-1}+Q^{-1}\left(\sum_{i=1}^{q} \widetilde{\Pi}_{i} \alpha_{i}^{-1}\right) Q^{-1} & Q^{-1}(\widehat{A}+\Delta \widehat{A}) & Q^{-1} J & Q^{-1} \widetilde{D} \\
\left(\widehat{A}^{\mathrm{T}}+\Delta \widehat{A}^{\mathrm{T}}\right) Q^{-1} & -Q^{-1} & 0 & 0 \\
J^{T} Q^{-1} & 0 & -Q^{-1} & 0 \\
\widetilde{D}^{\mathrm{T}} Q^{-1} & 0 & 0 & -W^{-1}
\end{array}\right]<0
$$


Rewrite $\widetilde{\Pi}_{i}$ in the following form:

$$
\widetilde{\Pi}_{i}=\left[\begin{array}{c}
\pi_{1 i} \\
\pi_{1 i}-\bar{\gamma} K \pi_{2 i}
\end{array}\right]\left[\begin{array}{c}
\pi_{1 i} \\
\pi_{1 i}-\bar{\gamma} K \pi_{2 i}
\end{array}\right]^{\mathrm{T}}+\left[\begin{array}{c}
0 \\
\sigma_{\gamma} K \pi_{2 i}
\end{array}\right]\left[\begin{array}{c}
0 \\
\sigma_{\gamma} K \pi_{2 i}
\end{array}\right]^{\mathrm{T}} .
$$

Using Schur Complement again, after some tedious calculation, we obtain that (53) is equivalent to the following matrix inequality

$$
\Upsilon=\left[\begin{array}{cc}
\Upsilon_{11} & \Upsilon_{12} \\
* & \Upsilon_{22}
\end{array}\right]<0
$$

where

$$
\begin{aligned}
& \Upsilon_{11}= {\left[\begin{array}{cccccc}
-R & 0 & R \breve{A} & 0 & 0 & 0 \\
* & -S & S \breve{A}-M & M-\bar{\gamma} N C & \sigma_{\tilde{\gamma}} N C & 0 \\
* & * & -R & 0 & 0 & \\
* & * & * & -S & 0 & 0 \\
* & * & * & * & -R & 0 \\
* & * & * & * & * & -S
\end{array}\right], } \\
& \Upsilon_{12}= \\
& \Upsilon_{22}= {\left[\begin{array}{ccccccc}
S \pi_{11} & \cdots & R \pi_{1 q} & 0 & \cdots & 0 & R B \\
S \pi_{11}-\bar{\gamma} N \pi_{21} & \cdots & S \pi_{1 q}-\bar{\gamma} N \pi_{2 q} & \sigma_{\gamma} N \pi_{21} & \cdots & \sigma_{\gamma} N \pi_{2 q} & S B-N D \\
0 & \cdots & 0 & 0 & \cdots & 0 & 0 \\
0 & \cdots & 0 & 0 & \cdots & 0 & 0 \\
0 & \cdots & 0 & 0 & \cdots & 0 & 0 \\
0 & \cdots & 0 & 0 & \cdots & 0 & 0
\end{array}\right], }
\end{aligned}
$$

with

$$
\begin{aligned}
& \breve{A}=(A+\Delta A), \\
& S G=M, \\
& S K=N .
\end{aligned}
$$

In order to eliminate the parameter uncertainty occurred in the system matrix, we rewrite (55) as follows:

$$
L+\hat{H} F \hat{E}+\hat{E}^{\mathrm{T}} F^{\mathrm{T}} \hat{H}^{\mathrm{T}}<0
$$

where

$$
\begin{aligned}
& L=\left[\begin{array}{cc}
\Psi_{11} & \Upsilon_{12} \\
* & \Upsilon_{22}
\end{array}\right], \\
& \hat{H}=\left[\begin{array}{lllllllllllll}
H^{\mathrm{T}} R & H^{\mathrm{T}} S & 0 & 0 & 0 & 0 & 0 & \cdots & 0 & 0 & \cdots & 0 & 0
\end{array}\right]^{\mathrm{T}} \text {, } \\
& \hat{E}=\left[\begin{array}{lllllllllllll}
0 & 0 & E & 0 & 0 & 0 & 0 & \cdots & 0 & 0 & \cdots & 0 & 0
\end{array}\right] \text {. }
\end{aligned}
$$

Now, applying Lemma 2 in [30] to (56), we know that (44) holds if and only if (51) holds. Moreover, noticing (48), we arrive at (40) from (44). Therefore, according to Corollary 1, the system (14) is exponentially meansquare stable, and the steady-state state covariance satisfies

$$
\widehat{Q} \leqslant Q .
$$

Since

$$
\widehat{Q}=\left[\begin{array}{cc}
X_{x x} & X_{x e} \\
X_{x e}^{\mathrm{T}} & X_{e e}
\end{array}\right] \text { and } Q=\left[\begin{array}{cc}
R^{-1} & 0 \\
0 & S^{-1}
\end{array}\right]
$$


we know that

$$
X_{e e} \leqslant S^{-1}
$$

Noticing that (45) implies

$$
S^{-1} \leqslant \widehat{X}^{-1}
$$

the requirement Q2) is also achieved. The proof is now complete.

Remark 4: The robust variance-constrained filtering problem has been solved for a class of nonlinear stochastic systems with missing measurements in terms of the feasibility of the LMIs (43)-(45) in Theorem 1. The LMIs can be solved efficiently via interior point method [1]. Note that LMIs (43)-(45) are affine in the scalar positive parameters $\varepsilon$ and $\alpha_{i}>0$. Hence, they can be defined as LMI variables in order to increase the possibility of the solutions and decrease conservatism with respect to the uncertainty $F$. Note that our main results are based on the LMI conditions. The LMI Control Toolbox implements state-of-the-art interiorpoint LMI solvers. While these solvers are significantly faster than classical convex optimization algorithms, it should be kept in mind that the complexity of LMI computations remains higher than that of solving, say, a Riccati equation. For instance, problems with a thousand design variables typically take over an hour on today's workstations. However, research on LMI optimization is a very active area in the applied math, optimization and the operations research community, and substantial speed-ups can be expected in the future.

Remark 5: It is worth pointing out that the main results in this paper can be easily extended to other more complicated systems, such as systems with multiple stochastic data packet losses, or stochastic systems with sector-bounded nonlinearity which is more general than that discussed in this paper. The results will appear in the near future.

\section{Numerical ExAmple}

In this section, we present an illustrative example to demonstrate the effectiveness of the proposed algorithms.

Consider the following discrete uncertain system with stochastic nonlinearities:

$$
\begin{aligned}
x_{k+1} & =\left(\left[\begin{array}{ccc}
-0.1 & 0.3 & -0.2 \\
0 & -0.25 & 0.1 \\
0.1 & 0 & 0.5
\end{array}\right]+\left[\begin{array}{c}
0.5 \\
0.6 \\
0
\end{array}\right] F_{k}\left[\begin{array}{lll}
0.8 & 0 & 0
\end{array}\right]\right) x_{k}+f\left(x_{k}\right)+\left[\begin{array}{c}
0.3 \\
0 \\
0.2
\end{array}\right] \omega_{k}, \\
y_{k} & =\gamma_{k}\left(\left[\begin{array}{lll}
1 & -0.6 & 2
\end{array}\right] x_{k}+g\left(x_{k}\right)\right)+\omega_{k} .
\end{aligned}
$$

where $F_{k}=\sin (0.6 k)$ is a deterministic perturbation matrix satisfying $F_{k} F_{k}^{\mathrm{T}} \leqslant I$, and $\omega_{k}$ is zero mean Gaussian white noise process with unity covariance. As mentioned in Remark 2, we now consider the stochastic nonlinearities $f\left(x_{k}\right)$ and $g\left(x_{k}\right)$ in the following three cases:

Case 1: $f\left(x_{k}\right)$ and $g\left(x_{k}\right)$ are nonlinearities with multiplicative noise of the following form:

$$
\begin{aligned}
& f\left(x_{k}\right)=a_{f} \sum_{i=1}^{n} \alpha_{f}^{i} x_{k}^{i} \xi_{k}^{i}, \\
& g\left(x_{k}\right)=a_{g} \sum_{i=1}^{n} \alpha_{g}^{i} x_{k}^{i} \xi_{k}^{i}, \quad i=1,2, \cdots, n,
\end{aligned}
$$

where $a_{f}$ and $a_{g}$ are known column vectors, $\alpha_{f}^{i}$ and $\alpha_{g}^{i}(i=1,2, \cdots, n)$ are known coefficients, $x_{k}^{i}$ is the $i$ th member of $x_{k}$, and $\xi_{k}^{i}$ are zero mean, uncorrelated Gaussian white noise processes with unity covariances. In 
this case, we assume

$$
\begin{aligned}
& f\left(x_{k}\right)=\left[\begin{array}{c}
0.2 \\
0.3 \\
0.5
\end{array}\right] \times\left(0.3 x_{k}^{1} \xi_{k}^{1}+0.4 x_{k}^{2} \xi_{k}^{2}+0.5 x_{k}^{3} \xi_{k}^{3}\right), \\
& g\left(x_{k}\right)=0.5 \times\left(0.3 x_{k}^{1} \xi_{k}^{1}+0.4 x_{k}^{2} \xi_{k}^{2}+0.5 x_{k}^{3} \xi_{k}^{3}\right) .
\end{aligned}
$$

Case 2: $f\left(x_{k}\right)$ and $g\left(x_{k}\right)$ are nonlinearities with the sign of a function:

$$
\begin{aligned}
& f\left(x_{k}\right)=b_{f} \sum_{i=1}^{n} \beta_{f}^{i} \cdot \operatorname{sign}\left(x_{k}^{i}\right) \cdot x_{k}^{i} \xi_{k}^{i}, \\
& g\left(x_{k}\right)=b_{g} \sum_{i=1}^{n} \beta_{g}^{i} \cdot \operatorname{sign}\left(x_{k}^{i}\right) \cdot x_{k}^{i} \xi_{k}^{i}, \quad i=1,2, \cdots, n .
\end{aligned}
$$

In this case, we assume

$$
\begin{aligned}
& f\left(x_{k}\right)=\left[\begin{array}{l}
0.2 \\
0.3 \\
0.5
\end{array}\right] \times\left(0.3 \cdot \operatorname{sign}\left(x_{k}^{1}\right) \cdot x_{k}^{1} \xi_{k}^{1}+0.4 \cdot \operatorname{sign}\left(x_{k}^{2}\right) \cdot x_{k}^{2} \xi_{k}^{2}+0.5 \cdot \operatorname{sign}\left(x_{k}^{3}\right) \cdot x_{k}^{3} \xi_{k}^{3}\right), \\
& g\left(x_{k}\right)=0.5 \times\left(0.3 \cdot \operatorname{sign}\left(x_{k}^{1}\right) \cdot x_{k}^{1} \xi_{k}^{1}+0.4 \cdot \operatorname{sign}\left(x_{k}^{2}\right) \cdot x_{k}^{2} \xi_{k}^{2}+0.5 \cdot \operatorname{sign}\left(x_{k}^{3}\right) \cdot x_{k}^{3} \xi_{k}^{3}\right) .
\end{aligned}
$$

Case 3: $f\left(x_{k}\right)$ and $g\left(x_{k}\right)$ are nonlinearities with the following form:

$$
\begin{aligned}
& f\left(x_{k}\right)=c_{f} \sum_{i=1}^{n} \rho_{f}^{i} x_{k}^{i}\left(\sin \left(x_{k}^{i}\right) \xi_{k}^{i}+\cos \left(x_{k}^{i}\right) \eta_{k}^{i}\right), \\
& g\left(x_{k}\right)=c_{g} \sum_{i=1}^{n} \rho_{g}^{i} x_{k}^{i}\left(\sin \left(x_{k}^{i}\right) \xi_{k}^{i}+\cos \left(x_{k}^{i}\right) \eta_{k}^{i}\right),
\end{aligned}
$$

where $\eta_{k}^{i}(i=1,2, \cdots, n)$ are zero mean Gaussian white noise processes with unity covariances which are mutually uncorrelated and also uncorrelated with $\xi_{k}^{i}$. In this case, we assume:

$$
\begin{aligned}
f\left(x_{k}\right) & =\left[\begin{array}{l}
0.2 \\
0.3 \\
0.5
\end{array}\right] \times\left(0.3 x_{k}^{1}\left(\sin \left(x_{k}^{1}\right) \xi_{k}^{1}+\cos \left(x_{k}^{1}\right) \eta_{k}^{1}\right)\right. \\
& \left.+0.4 x_{k}^{2}\left(\sin \left(x_{k}^{2}\right) \xi_{k}^{2}+\cos \left(x_{k}^{2}\right) \eta_{k}^{2}\right)+0.5 x_{k}^{3}\left(\sin \left(x_{k}^{3}\right) \xi_{k}^{3}+\cos \left(x_{k}^{3}\right) \eta_{k}^{3}\right)\right) \\
g\left(x_{k}\right) & =0.5 \times\left(0.3 x_{k}^{1}\left(\sin \left(x_{k}^{1}\right) \xi_{k}^{1}+\cos \left(x_{k}^{1}\right) \eta_{k}^{1}\right)+0.4 x_{k}^{2}\left(\sin \left(x_{k}^{2}\right) \xi_{k}^{2}+\cos \left(x_{k}^{2}\right) \eta_{k}^{2}\right)\right. \\
& \left.+0.5 x_{k}^{3}\left(\sin \left(x_{k}^{3}\right) \xi_{k}^{3}+\cos \left(x_{k}^{3}\right) \eta_{k}^{3}\right)\right) .
\end{aligned}
$$

Now, we can easily check all the above three classes of stochastic nonlinearities satisfy the following equality:

$$
\begin{aligned}
& \mathbb{E}\left\{\left[\begin{array}{l}
f\left(x_{k}\right) \\
g\left(x_{k}\right)
\end{array}\right]\left[f^{\mathrm{T}}\left(x_{k}\right) g^{\mathrm{T}}\left(x_{k}\right)\right] \mid x_{k}\right\} \\
& =\left[\begin{array}{llll}
0.04 & 0.06 & 0.10 & 0.10 \\
0.06 & 0.09 & 0.15 & 0.15 \\
0.10 & 0.15 & 0.25 & 0.25 \\
0.10 & 0.15 & 0.25 & 0.25
\end{array}\right] x_{k}^{\mathrm{T}}\left[\begin{array}{ccc}
0.09 & 0 & 0 \\
0 & 0.16 & 0 \\
0 & 0 & 0.25
\end{array}\right] x_{k} .
\end{aligned}
$$


Let the stochastic variable $\gamma_{k} \in \mathbb{R}$ be a Bernoulli distributed white sequence taking values on 0 and 1 with $\operatorname{Prob}\left\{\gamma_{k}=1\right\}=\mathbb{E}\left\{\gamma_{k}\right\}=0.9$. Hence, $\sigma_{\gamma}=\sigma_{\tilde{\gamma}}=0.3$.

Choosing $\sigma_{1}^{2}=0.5, \sigma_{2}^{2}=0.5, \sigma_{3}^{2}=0.8$ as the estimation error variances upper bounds, we employ Matlab Toolbox to find the desired filter parameters by using Theorem 1 and obtain

$$
\begin{aligned}
M & =\left[\begin{array}{ccc}
-0.6109 & 0.0877 & 0.4967 \\
0.0877 & -0.6543 & -0.3931 \\
0.4967 & -0.3931 & 1.9735
\end{array}\right], \quad N=\left[\begin{array}{c}
2.1466 \\
-0.2336 \\
0.9600
\end{array}\right], \\
R & =\left[\begin{array}{ccc}
5.6595 & -1.3826 & -0.2050 \\
-1.3826 & 5.5182 & -1.2893 \\
-0.2050 & -1.2893 & 4.5803
\end{array}\right], \quad S=\left[\begin{array}{ccc}
5.3355 & -1.6377 & 0.4468 \\
-1.6377 & 4.7606 & -1.1655 \\
0.4468 & -1.1655 & 4.2440
\end{array}\right] . \\
\alpha_{1} & =11.0146, \quad \alpha_{2}=10.5435, \quad \alpha_{3}=9.4927, \quad \varepsilon=6.6309 .
\end{aligned}
$$

Finally, the obtained filter parameters are calculated as follows:

$$
G=\left[\begin{array}{ccc}
-0.1230 & -0.0274 & 0.0712 \\
0.0085 & -0.1810 & 0.0578 \\
0.1323 & -0.1395 & 0.4734
\end{array}\right], \quad K=\left[\begin{array}{c}
0.4308 \\
0.1537 \\
0.2231
\end{array}\right]
$$

The simulation results are shown in Figs. 1-6. The filtering error variances of the states $x_{k}^{1}, x_{k}^{2}$ and $x_{k}^{3}$ for Case 1- Case 3 are given in Figs. 1-3. Fig. 4 shows the actual state responses $x_{k}^{1}$ and its estimate $\hat{x}_{k}^{1}$ for Case 1 , while Fig. 5 shows the actual state responses $x_{k}^{2}$ and its estimate $\hat{x}_{k}^{2}$ for Case 3 . From Fig. 6 we could easily see that, different data missing rates will cause different performances on filtering error variance. To be specific, the filtering error variance will become larger when the data missing is severe, which is reasonable and understandable.

\section{Conclusion}

In this paper, a robust variance-constrained filter has been designed for a class of nonlinear stochastic systems with both parameter uncertainties and probabilistic missing measurements. A general framework for solving this problem has been established using an LMI approach. Sufficient conditions have been derived in terms of a set of feasible LMIs. An illustrative numerical example has been provided to demonstrate the usefulness and effectiveness of the proposed approach. It is worth pointing out that the main results developed in this paper could be applied to many engineering problems, for example, the maneuvering target tracking problem that is an important branch of signal processing. Due to the complicated working conditions and the limited capacity of data transmission, it might be the case from time to time that the measurements may contain noise only. Therefore, the robust filtering problem for stochastic nonlinear systems with multiple missing measurements becomes an important topic of research. By using the algorithm developed in this paper, the filtering problem with missing measurements could be dealt with conveniently within the LMI framework.

\section{REFERENCES}

[1] S. Boyd, L.E. Ghaoui, E. Feron, V. Balakrishnan, Linear Matrix Inequalities in System and Control Theory, SIAM Stud. Appl. Math., Philadelphia, 1994.

[2] M.F. Bugallo, S. Xu, Performance comparison of EKF and particle filtering methods for maneuvering targets, Digital Signal Processing, 17 (4) (2007) 774-786.

[3] K.Y. Chang, W.J. Wang, $H_{\infty}$ norm constraints and variance control for stochastic uncertain large-scale systems via the siliding mode concept, IEEE Trans. Circuits. Syst. Fundamental Theory and Applications, 46 (10) (1999) 1275-1280.

[4] C. Chen, H. Liu and X. Guan, $H_{\infty}$ filtering of time-delay T-S fuzzy systems based on piecewise Lyapunov-Krasovskii functional, Signal Processing, 89 (10) (2009) 1998-2005. 
[5] E.G.Jr. Collins, R.E. Skelton, A theory of state covariance assignment for discrete systems, IEEE Trans. Automat. Contr., 32 (1) (1987) 35-41.

[6] O. Costa, Stationary filter for linear minimum mean square error estimator of discrete-time markovian jump systems, IEEE Trans. Automat. Contr., 48 (8) (2002) 1351-1356.

[7] H. Dong, Z. Wang and H. Gao, $H_{\infty}$ filtering for systems with repeated scalar nonlinearities under unreliable communication links, Signal Processing, 89 (8) (2009), 1567-1575.

[8] S. Esfahani, I. Peterson, An LMI approach to output-feedback-guaranteed cost control for uncertain time-delay systems, International Journal of Robust and Nonlinear Control, 10 (2000) 157-174.

[9] P. Gahinet, Explicit controller formulas for LMI based $H_{\infty}$ synthesis, Automatica, 32 (1996) $1007-1014$.

[10] H. Gao, J. Lam, L. Xie, C. Wang, New approach to mixed $H_{2} / H_{\infty}$ filtering for polytopic discrete-time systems, IEEE Trans. Signal Processing, 53 (8) (2005) 3183-3192.

[11] H. Gao, C. Wang, A delay-dependent approach to robust $H_{\infty}$ filtering for uncertain discrete-time state-delayed systems, IEEE Trans. Signal Processing, 52 (6) (2004) 1631-1640.

[12] H. Gao, Y. Zhao, J. Lam and K. Chen, $H_{\infty}$ fuzzy filtering of nonlinear systems with intermittent measurements, IEEE Trans. Fuzzy Systems, 17 (2) (2009) 291-300.

[13] H. Gao and T. Chen, $H_{\infty}$ estimation for uncertain systems with limited communication capacity, IEEE Trans. Automatic Control, 52 (11) (2007) 2070-2084.

[14] E. Gershon, U. Shaked, Static $H_{2}$ and $H_{\infty}$ output-feedback of discrete-time LTI systems with state multiplicative noise, Syst. 6 Contr. Letters 55 (2006) 232-239.

[15] M. Hadidi and S. Schwartz, Linear recursive state estimators under uncertain observations, IEEE Trans. Automat. Contr., 24 (6) (1979) 944-948.

[16] C. Han and H. Zhang, Linear optimal filtering for discrete-time systems with random jump delays, Signal Processing, 89 (6) (2009) 1121-1128.

[17] Y. He, G.P. Liu, D. Rees and M. Wu, $H_{\infty}$ filtering for discrete-time systems with time-varying delay, Signal Processing, 89 (3) (2009) 275-282.

[18] A.F. Hotz, R.E. Skelton, A covariance control theory, Int. J. Control, 46 (1) (1987) 13-32.

[19] Y. S. Hung, F. Yang, Robust $H_{\infty}$ filtering with error variance constraints for uncertain discrete time-varying systems with uncertainty, Automatica, 39 (7) (2003) 1185-1194.

[20] J. Linares-Perez, A. Hermoso-Carazo, R. Caballero-Aguila, J.D. Jimenez-Lopez, Least-squares linear filtering using observations coming from multiple sensors with one- or two-step random delay, Signal Processing, 89 (10) (2009), $2045-2052$.

[21] Q. Ling and M.D. Lemmon, Optimal dropout compensation in networked control systems, in Proc. IEEE Conf. Decision Control, Hawaii, Dec. 2003 670C675.

[22] N. Nahi, Optimal recursive estimation with uncertain observation, IEEE Trans. Inf. Theory, IT-15 (4) (1969) 457-462.

[23] W. NaNacara, E. Yaz, Recursive estimator for linear and nonlinear systems with uncertain observations, Signal Process, 62 (1997) 215-228.

[24] A.V. Savkin, I.R.Petersen, S.O.R.Moheinami, Model validation and state estimation for uncertain continuous-time systems with missing discrete-continuous data, Comput. Elect. Eng., 25 (1) (1999) 29-43.

[25] P. Shi, E.K. Boukas, Y. Shi, R.K. Agarwal, Optimal guaranteed cost control of uncertain discrete time-delay systems, J. Comput. Appl. Math., 157 (2) (2003) 435-451

[26] A. Subramanian, A. H. Sayed, Multiobjective filter design for uncertain stochastic time-dealy systems, IEEE Trans. Automat. Contr., 49 (1) (2004) 149-154.

[27] T.J. Tarn, Y. Rasis, Observers for nonlinear stochastic systems, IEEE Trans. Automat. Contr., 21 (6) (1976) $441-447$.

[28] Z. Wang, D.W.C. Ho, X. Liu, Variance-constrained filtering for uncertain stochastic systems with missing measurements, IEEE Trans. Automat. Contr., 48 (7) (2003) 560-567.

[29] Z. Wang, F. Yang, D.W.C. Ho, X. Liu, Robust finite-horizon filtering for stochastic systems with missing measurements, IEEE Trans. Signal Processing, 12 (6) (2005) 137-440.

[30] Z. Wang, F. Yang, D.W.C. Ho, X. Liu, Robust $H_{\infty}$ filtering for stochastic time-delay systems with missing measurements, IEEE Trans. Signal Processing, 54 (7) (2006) 2579-2567.

[31] L. Wu and Z. Wang, Fuzzy filtering of nonlinear fuzzy stochastic systems with time-varying delay, Signal Processing, 89 (9) (2009), 1739-1753.

[32] L. Xie, Y.C. Soh, C.E.de Souza, Robust Kalman filtering for uncertain discrete time systems, IEEE Trans. Automat. Contr., 39 (1994) 1310-1314.

[33] L. Xie, C.E.de Souza, Robust control for linear systems with norm-bounded time-varying uncertainty, IEEE Trans. Automat. Contr., 37 (1992) 1188-1191.

[34] F. Yang, Z. Wang, D.W.C. Ho, X. Liu, Robust $H_{2}$ Filtering for a Class of systems with stochastic Nonlinearities, IEEE Trans. Circuits Syst. II: Express Briefs, 53 (3) (2006) 235-239 
[35] K. Yasuda, S. Kherat, R.E. Skelton, E. Yaz, Covariance control and robustness of bilinear systems, in: Proc. IEEE Conf. Decision Contr., Honolulu, Hawaii, 1990, pp. 1421-1425.

[36] Y. Yaz, E. Yaz, On LMI formulations of some problems arising in nonlinear stochastic system analysis, IEEE Trans. Automat. Contr., 44 (4) (1999) 813-816.

[37] E. Yaz, Robust design of stochastic controllers for nonlinear systems, IEEE Trans. Automat. Contr., 34 (3) (1989) 349-353.

Filtering error variances

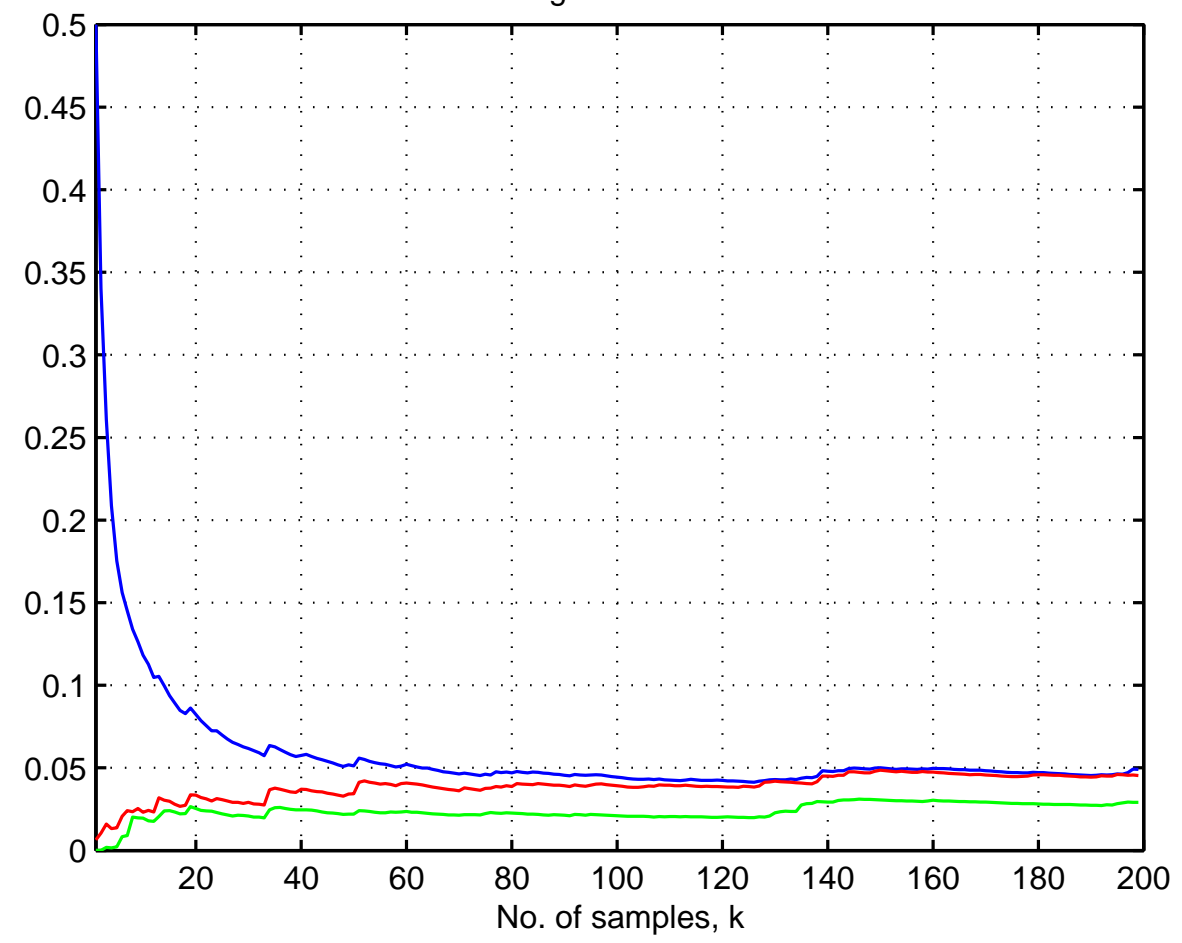

Fig. 1. The filtering error variances for Case 1. 


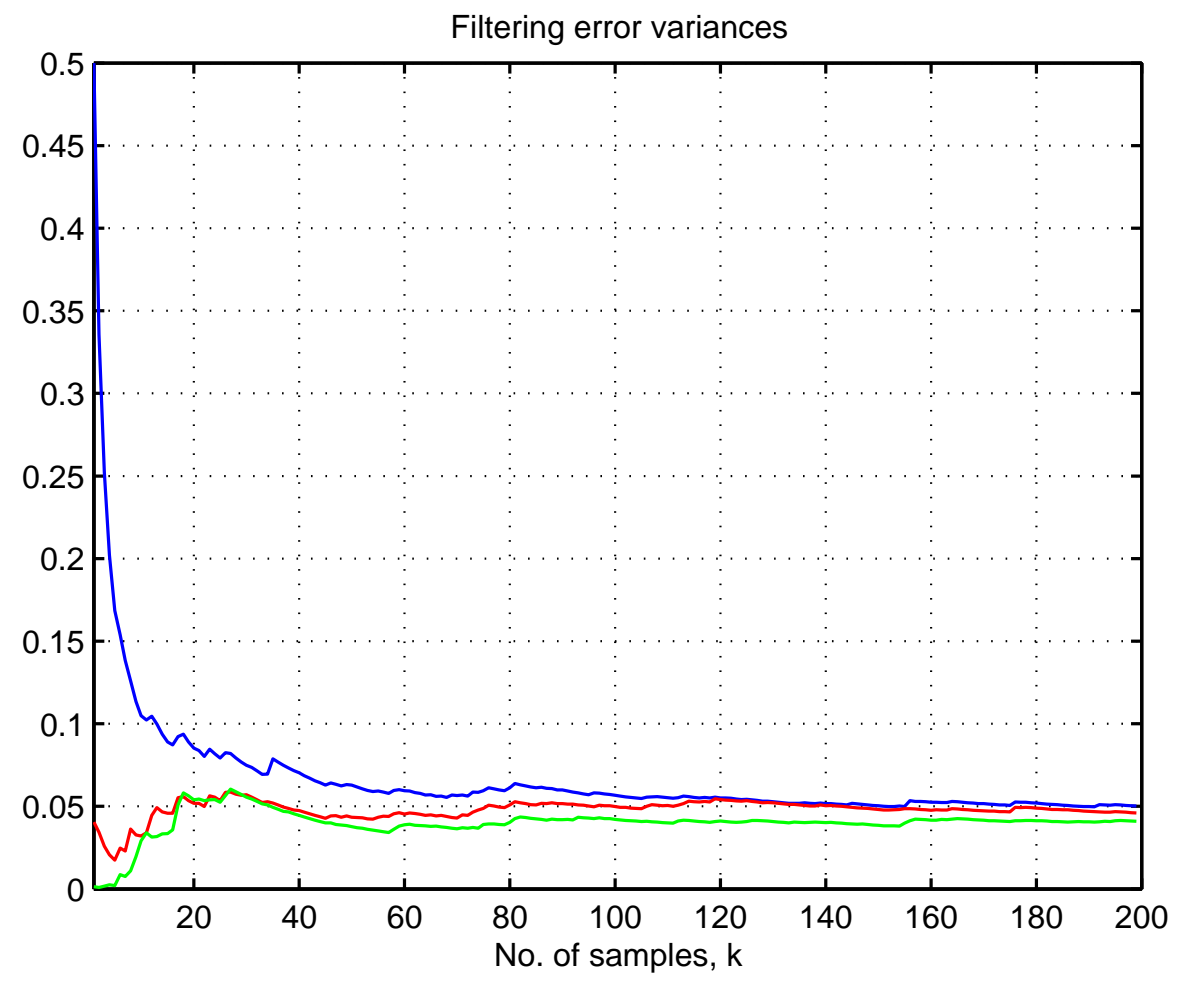

Fig. 2. The filtering error variances for Case 2 .

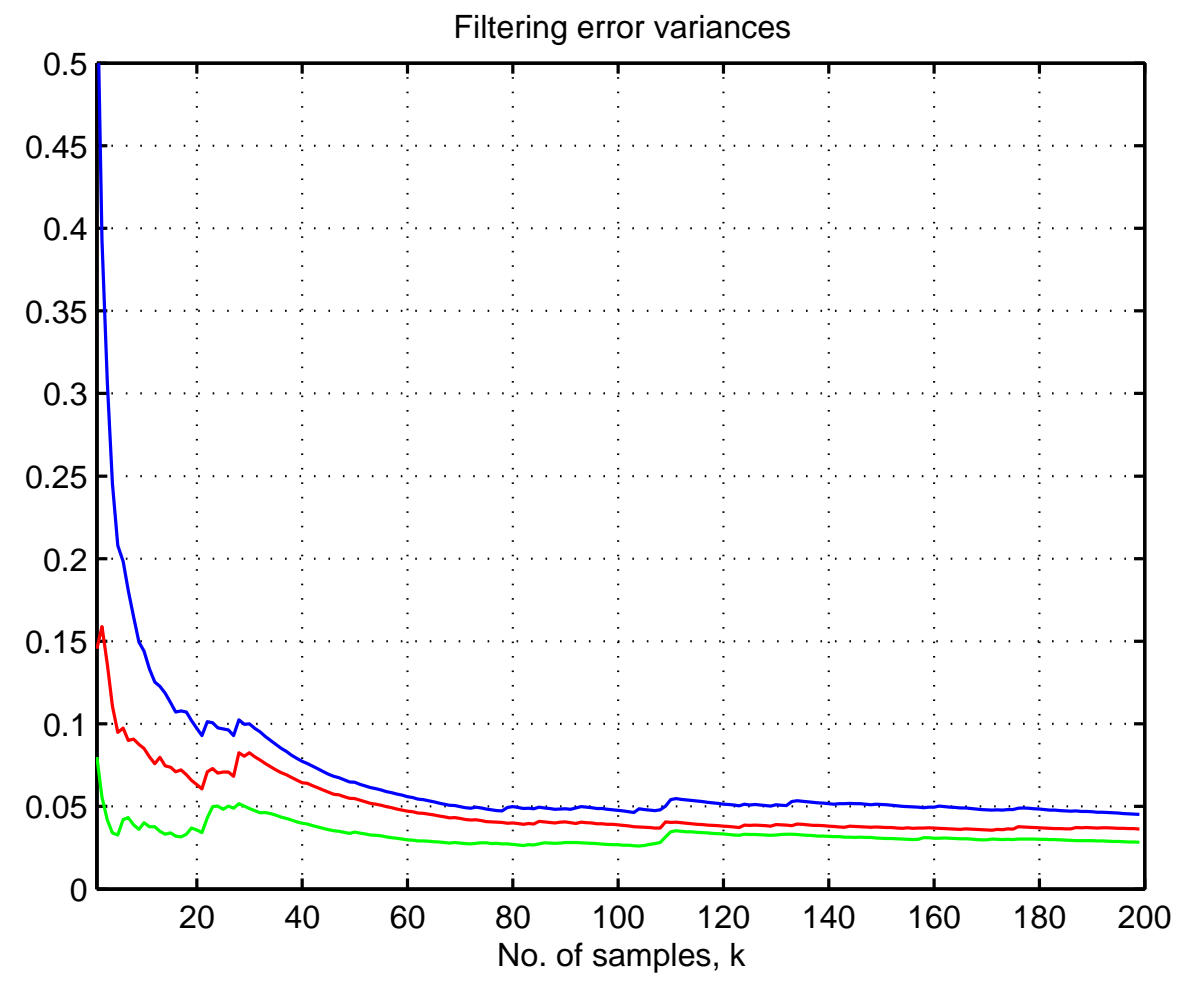

Fig. 3. The filtering error variances for Case 3 . 


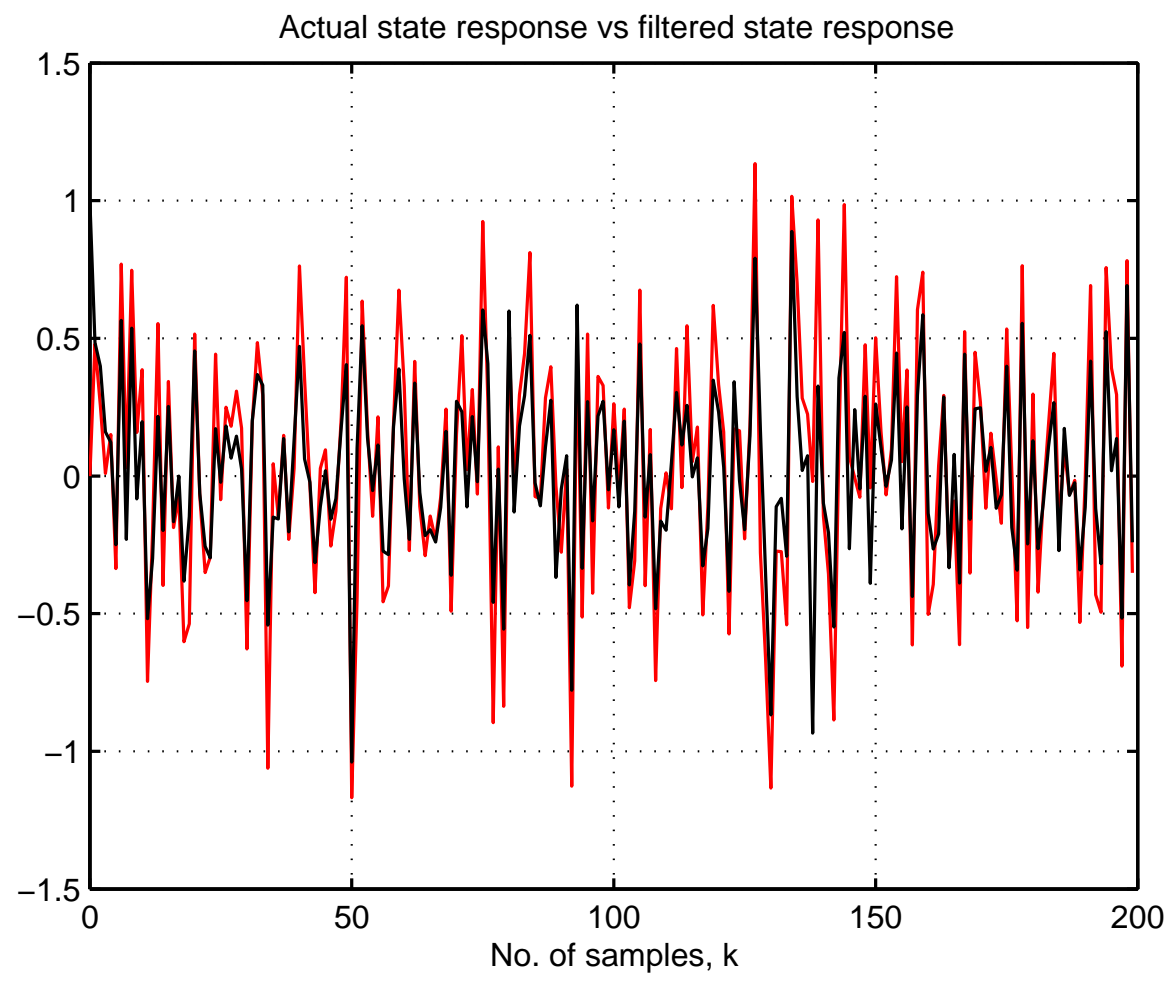

Fig. 4. The actual state $x_{k}^{1}$ (black) and its estimate $\hat{x}_{k}^{1}$ for Case 1 .

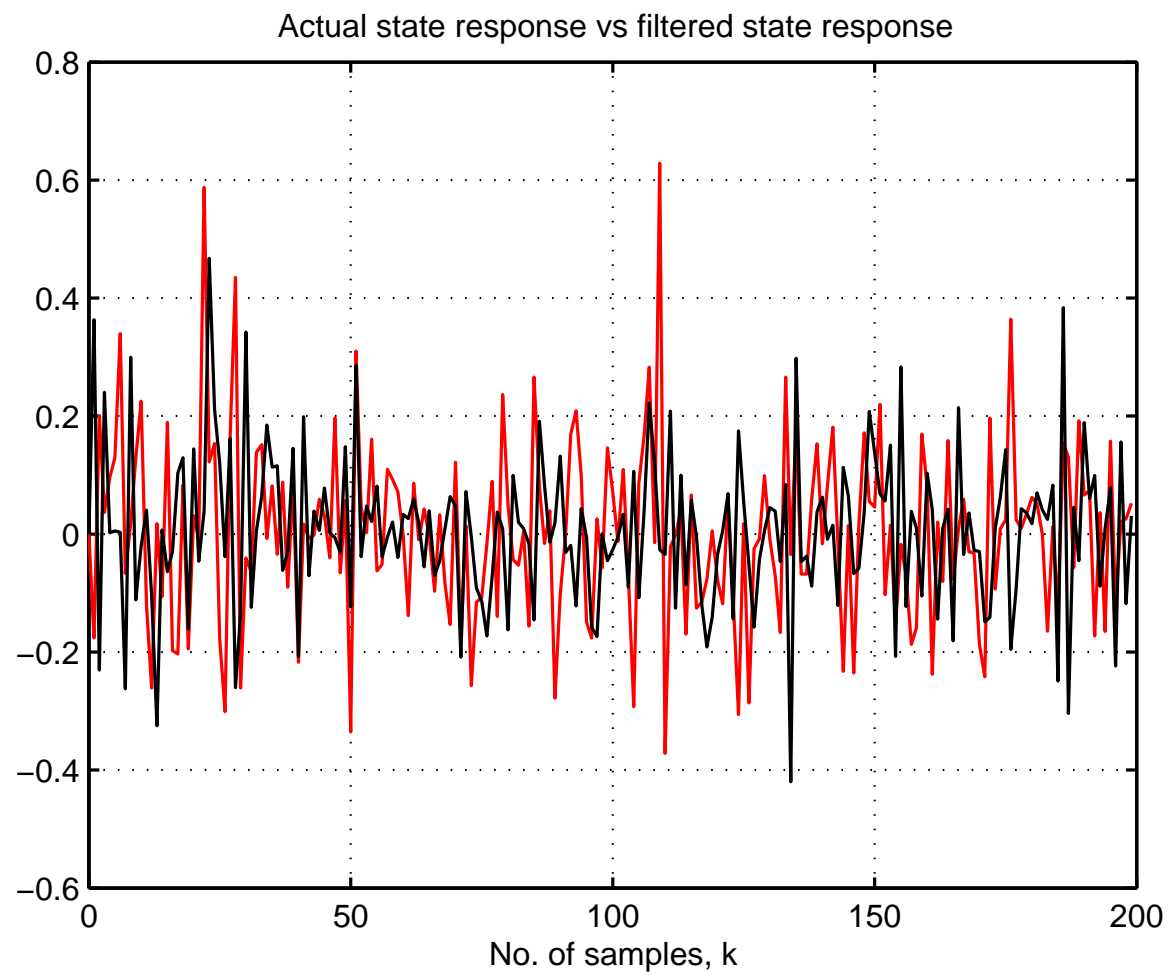

Fig. 5. The actual state $x_{k}^{2}$ (black) and its estimate $\hat{x}_{k}^{2}$ for Case 3 . 


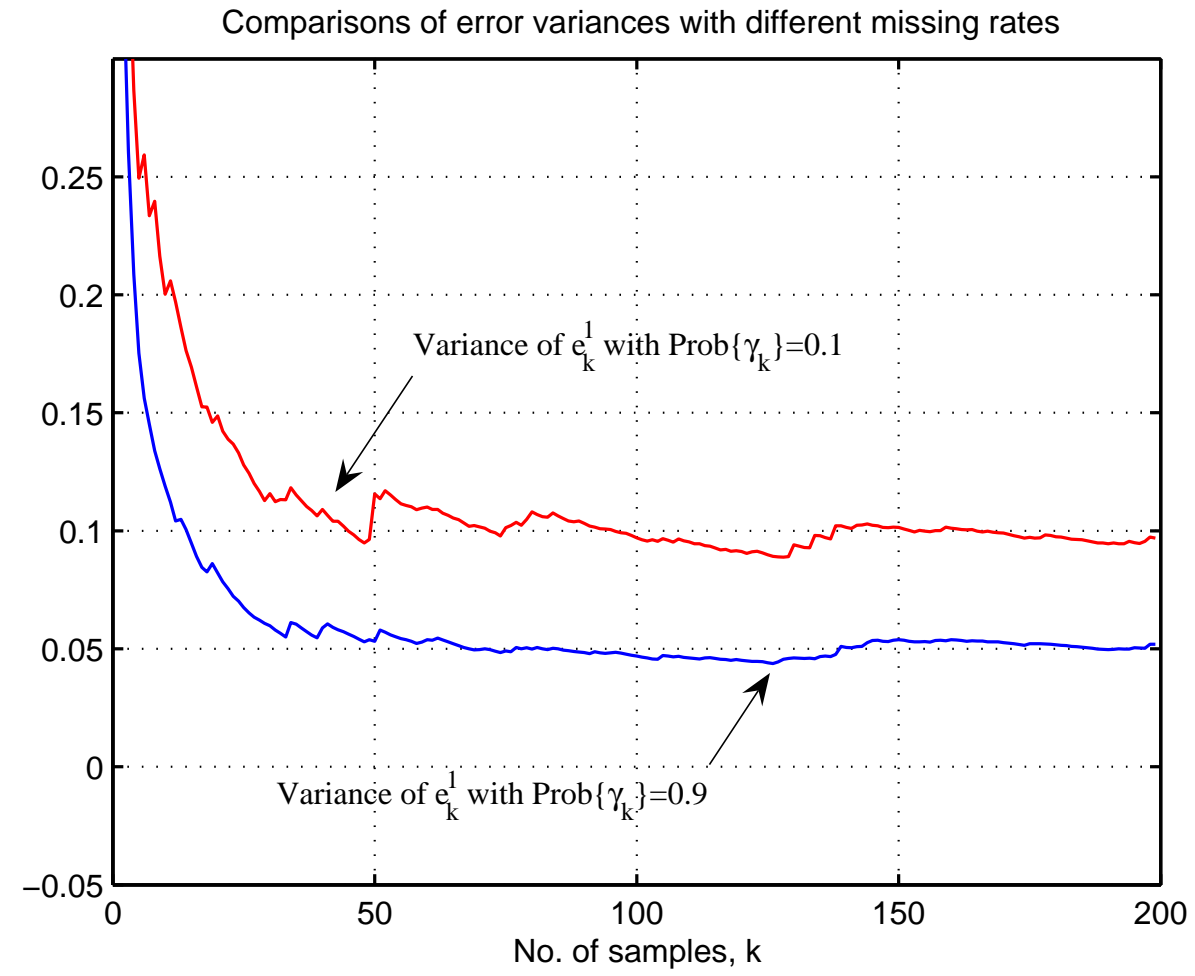

Fig. 6. The comparison between filtering error variance of different data missing rates. 